\title{
Visualization of single-walled carbon nanotubes electrical networks by scanning force microspy
}

Cite as: Appl. Phys. Lett. 79, 2979 (2001); https://doi.org/10.1063/1.1414301

Submitted: 28 June 2001 • Accepted: 04 September 2001 • Published Online: 23 October 2001

P. J. de Pablo, C. Gómez-Navarro, A. Gil, et al.

ARTICLES YOU MAY BE INTERESTED IN

Performing current versus voltage measurements of single-walled carbon nanotubes using scanning force microscopy

Applied Physics Letters 80, 1462 (2002); https://doi.org/10.1063/1.1453475

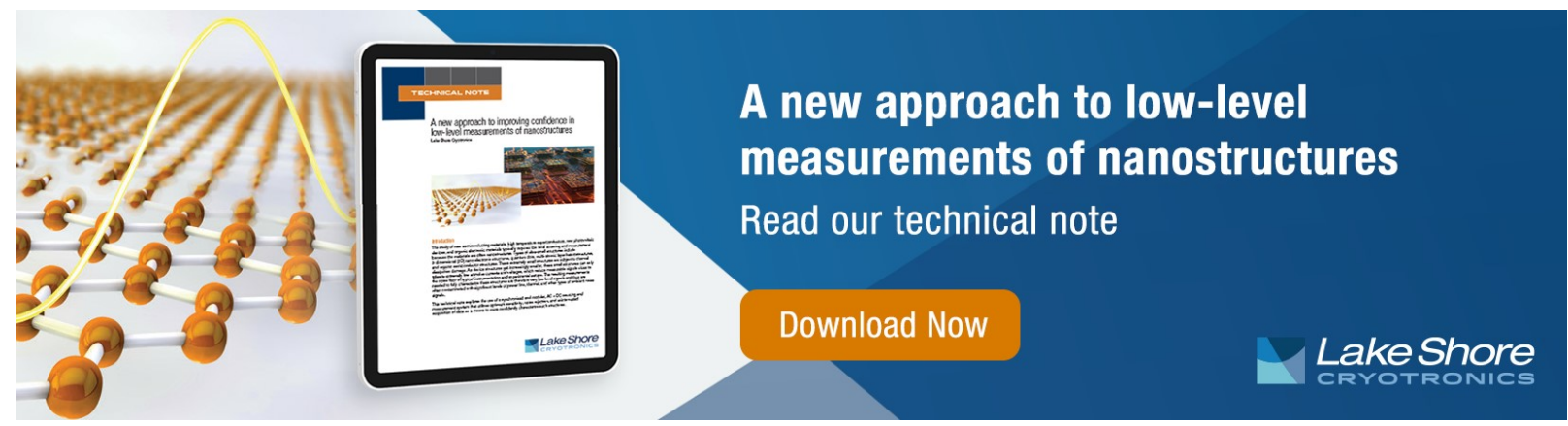




\title{
Visualization of single-walled carbon nanotubes electrical networks by scanning force microspy
}

\author{
P. J. de Pablo, C. Gómez-Navarro, A. Gil, and J. Colchero \\ Laboratorio de Nuevas Microscopías, Departamento de Física de la Materia Condensada, Universidad \\ Autónoma de Madrid, E-28049 Madrid, Spain \\ M. T. Martinez, A. M. Benito, and W. K. Maser \\ Instituto de Carboquímica, CSIC María de Luna 12, E-50015 Zaragoza, Spain \\ J. Gómez-Herrero a) and A. M. Baró \\ Laboratorio de Nuevas Microscopías, Departamento de Física de la Materia Condensada, Universidad \\ Autónoma de Madrid, E-28049 Madrid, Spain
}

(Received 28 June 2001; accepted for publication 4 September 2001)

\begin{abstract}
In this letter, a method is presented to qualitatively investigate the electrical connection between single-walled carbon nanotubes and gold electrodes. By applying a time independent bias voltage to the scanning force microscope tip, there is a clear apparent growth effect in the topography of the electrically connected carbon nanotubes. This effect is induced by the electrostatic interaction of the tip with the free charges located at the conducting areas of the surface. This is a general method that could be applied to any molecular conducting nanowire. (C) 2001 American Institute of Physics. [DOI: 10.1063/1.1414301]
\end{abstract}

In recent years it has been an increasing interest for the molecular wires, ${ }^{1}$ principally carbon nanotubes. ${ }^{2}$ A carbon nanotube is a graphene layer rolled up forming a tube of nanometer size. They have been found to be good electrical conductors with astonishing properties, showing ballistic, ${ }^{3}$ superconducting, ${ }^{4}$ and Aharonov-Bohm effects. ${ }^{5}$ Carbon nanotubes have been also used as field emission emitters, ${ }^{6}$ chemical detectors, ${ }^{7}$ nanotweezers, ${ }^{8}$ etc. Some theoretical work has been undertaken to explain the overlap between the metallic electrodes and the carbon nanotubes. ${ }^{9}$ In addition, a number of contributions have been published on the electrical transport properties of carbon nanotubes when a current flows trough them. ${ }^{10,11}$ On the contrary, only a few works have been done on the electrostatic forces, which are originated by these nanowires. From the very beginning, electrostatic interaction has been known to play a central role in the scanning force microscopy (SFM) experiments. ${ }^{12}$ Bachtold et $a l .{ }^{13}$ report electrostatic characterization of single-walled nanotubes while a direct current (dc) flows. From that work the electron correlation length is obtained. In this letter we present a method based upon the electrostatic interaction between tip and sample to detect electrical connected nanotubes without flowing current. This should be a general method to test the electrical connectivity of any molecular wire.

Samples are prepared by adsorbing single walled carbon nanotubes (SWNT) on a $\mathrm{SiO}_{2}$ substrate. ${ }^{14}$ After deposition of the molecules a thermal gold evaporation is performed. The final result is a sample with two gold electrodes separated by a thin line $(\sim 5 \mu \mathrm{m})$ free of gold with a random distribution of molecules. ${ }^{15}$ Some of the molecules appear partially covered with gold. A similar setup has been used with multiwalled carbon nanotubes elsewhere. ${ }^{16}$ The aim of this work

${ }^{a)}$ Electronic mail: julio.gomez@uam.es is to discriminate between the molecules contacting the gold electrode and the isolated molecules in Fig. 1.

SFM is used in noncontact dynamic mode. ${ }^{17}$ Briefly, in this mode the tip is oscillated at its resonance frequency, and the feedback loop is engaged to a fixed value of the amplitude of the cantilever. In this way topography images of the surface can be obtained. To be sensitive to the electrostatic forces, Olympus cantilevers with $80 \mathrm{kHz}$ resonance frequency consecutively covered with titanium and gold are used.

Figure 2(a) shows a SFM topography image of the electrode border with several SWNT connected to the gold with a bias $V=0 \mathrm{~V}$ applied to the SFM tip. A label has been added to mark the position of some relevant nanotubes. In Fig. 2(b) a bias $V=2 \mathrm{~V}$ is applied to the SFM tip. As a consequence of

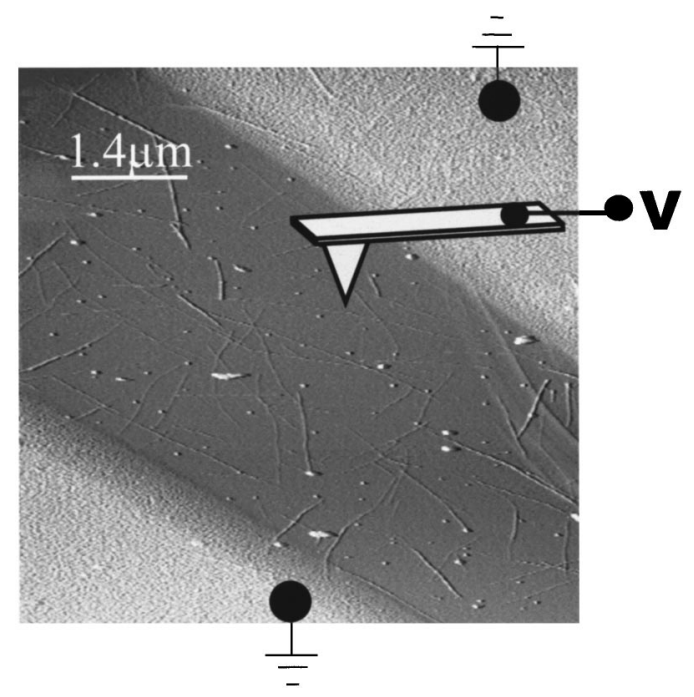

FIG. 1. Experimental setup. A region of $\mathrm{SiO}_{2}$ surrounded by two gold electrodes is prepared, so a random population of single walled carbon nanotubes connected to the electrodes is obtained. 

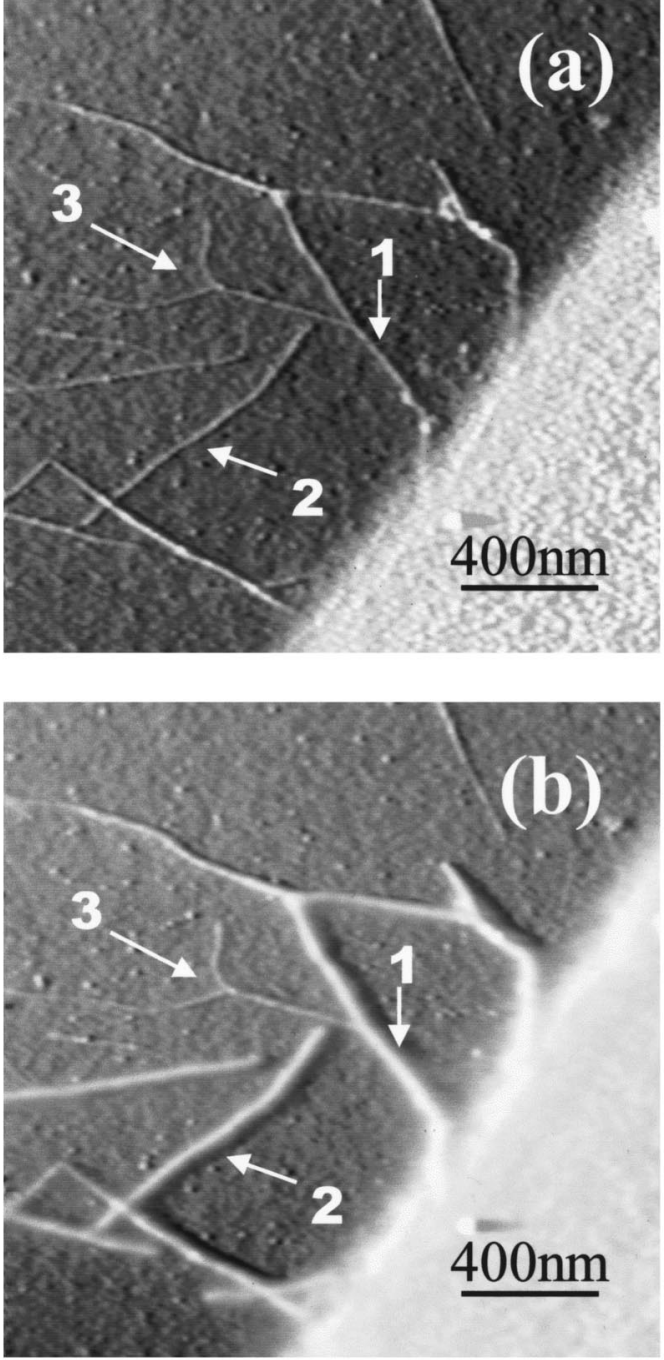

FIG. 2. (a) Shows an area with SWNTs connected and disconnected to the gold while a voltage of $0 \mathrm{~V}$ is applied to the SFM tip. The topography image has been taken using noncontact dynamic SFM mode. (b) A voltage of $2 \mathrm{~V}$ is applied to the tip in the same area of (a). As a consequence the topography image shows an increment of the height and blurring effects on the areas of the surface connected to ground. Labels 1, 2, and 3 mark to different nanotubes: directly connected to the gold (1), a nanotube connected via other nanotube (2), and a disconnected nanotube (3).

the electrostatic interaction, the topography image shows an blurring effect of the areas of the surface connected to ground, i.e., the gold electrode and the connected nanotubes. Specifically, the nanotubes connected to the electrode show a higher and wider topography than that those in Fig. 2(a). Directly connected nanotubes to the gold [nanotube 1 in Fig. 2(b)] and nanotubes connected to the gold via a second or even a third nanotube [nanotube 2 in Fig. 2(b)] show this blurring effect. Another case is that the electrically isolated nanotubes [like nanotube 3 in Fig. 2(b)] do not show this effect.

The electrostatic force between the tip and the connected nanotubes can explain this effect. The voltage applied to the tip induces the charges to flow from the ground to the nanotubes to screen the effect of the electrostatic field generated by the tip. This does not happen in the electrically isolated areas of the sample, like the $\mathrm{SiO}_{2}$ substrate or the isolated SWNT, as the nanotube 3 of Fig. 2(b).

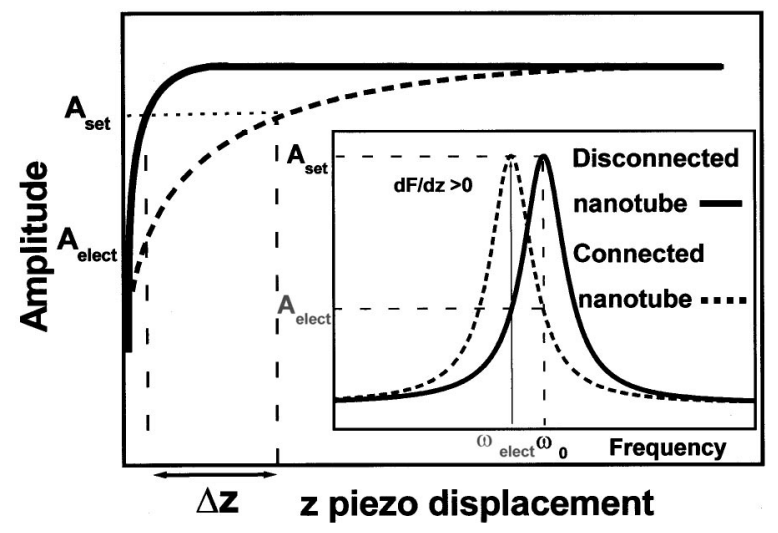

FIG. 3. Behavior of the oscillating amplitude vs. $z$ piezoelectric displacement for insulating areas (solid) and ground connected areas (dashed). The inset shows the change of the resonance frequency with the electrostatic interaction at a fixed distance from the sample. When a voltage is applied to the tip, the solid chart is the amplitude on the insulator regions where the resonance peak is centered at $\omega_{0}$. The dashed chart is the amplitude on connected nanotubes. Here the resonance curve is shifted due to the electrostatic interaction. When the system is driven at a fixed frequency the amplitude changes from $A_{\text {set }}$ to $A_{\text {elect }}$. As a consequence the feedback loop moves the tip away from the sample to get $A_{\text {set }}$ again.

Let us explain qualitatively how this effect is due to the electrostatic forces between connected nanotubes and the tip. The oscillation of the tip under the electrostatic force can be modeled by:

$$
m \frac{d^{2} z}{d t^{2}}=-k z-\frac{m \omega_{0}}{Q} \frac{d z}{d t}+F_{0} \cos (\omega t)+F_{e}(z)
$$

where $z$ is the distance between tip and sample, $m$ is the effective cantilever mass, $k$ is the force constant, $\omega_{0}$ the resonance frequency of the system, $Q$ the quality factor, $F_{0} \cos (\omega t)$ the cantilever driving force, and $F_{e}$ the electrostatic force between tip and sample. ${ }^{18}$ In the case of absence of $F_{e}{ }^{19}$ the resonance peak of the amplitude is centered at $\omega_{0}$, the resonance frequency of the free system (see inset of Fig. 3). When $V_{\text {tip }} \neq 0$ and $F_{e}$ is assumed to be linear, ${ }^{20}$ the resonance frequency changes by $\Delta \omega$ :

$$
\frac{\Delta \omega}{\omega_{0}} \approx\left(\frac{1}{2 k}\right) \frac{d F_{e}}{d z},
$$

where $\Delta \omega$ is negative because electrostatic forces are attractive. Let us see how this can induce changes in the topography images. When $F_{e}$ is not present between tip and sample, the amplitude at which the topography images are taken is $A_{\text {set }}$ (see Fig. 3). But when $F_{e}$ is present the amplitude decreases to $A_{\text {elect }}$ because of the change of the resonance frequency (see inset of Fig. 3). As a consequence the feedback loop moves the tip away from the surface to keep the amplitude constant. ${ }^{21}$ In this way the electrostatic force is originating an extra height in the topography, which is seen in Fig. 2(b). This extra height depends not only on the tip voltage, but also on the feedback parameters and amplitude set point. Using an appropriate data processing algorithm, quantitative information could be also obtained. ${ }^{22}$

In Fig. 4 a network of connected nanotubes is shown. Notice that only the nanotubes labeled with 1 and 2 are not electrically connected to the gold electrode. The rest of the nanotubes are connected to the gold electrode by themselves or via another nanotube. If a current versus voltage charac- 


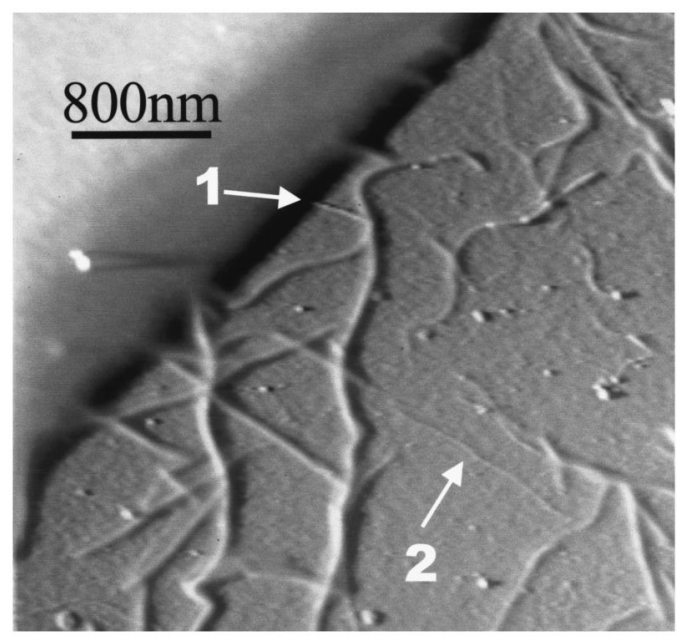

FIG. 4. Electrical nanotube network. Only nanotubes 1 and 2 are not electrically connected to the gold. A voltage of $1.6 \mathrm{~V}$ is applied to the tip. The SWNT network connected to the electrode can be clearly seen.

teristic is performed by contacting the metal tip with a SWNT directly connected with the gold electrode, a current flows through the molecule. If the same experiment is performed with a SWNT connected to the gold electrode via a second molecule the experiment always shows a much larger resistance that can be in the $G \Omega$ range or even larger. This high resistance is due to the molecule-molecule contact. Nevertheless, those nanotubes also present a clear blurring effect. We conclude that this measurement is fairly insensible to the molecule resistance and hence, this method can be applied in molecules with a much higher resistivity than SWNT. Another possibility is to distinguish metallic from semiconducting nanotubes. ${ }^{23}$

Up to now we have applied this method to SWNT connected to one electrode. A natural extension should be to check the electrical connection of a molecule with two electrodes simultaneously. To do so, consecutive images of a selected nanotube should be taken grounding one electrode, leaving the second one floating, and viceversa.

In summary, we have shown an easy method to visualize electrical networks of SWNT by means of the SFM. The connected nanotubes show a blurring effect in the topography when a dc voltage is applied to the metallic tip. This effect can be easily explained by the electrostatic forces between the tip and the charges that flow from the ground to the connected SWNT, induced by the electrostatic field of the tip. This method could be used to check the reliability of future SWNT circuits of the incoming carbon electronics as well as other nanowires.

The authors acknowledge fruitful discussions with P. A. Serena and J. J. Sáenz. Financial support has been provided by Comunidad de Madrid through Project No. 07N/0024/ 1998 and Ministerio de Educación y Ciencia through Project No. PB95-0169.

${ }^{1}$ S. Datta, Electron Transport in Mesoscopic Systems (Cambridge University Press, Cambridge, UK, 1995).

${ }^{2}$ S. Iijima, Nature (London) 354, 56 (1991).

${ }^{3}$ S. Frank, P. Poncharal, Z. L. Wang, and W. A. de Heer, Science 280, 1744 (1998).

${ }^{4}$ A. Yu. Kasumov, R. Deblock, M. Kociak, B. Reulet, H. Bouchiat, I. I. Khodos, Yu. B. Gorbatov, V. T. Volkov, C. Journet, and M. Burghard, Science 284, 1508 (1999).

${ }^{5}$ A. Bachtold, C. Strunk, J.-P. Salvetat, J.-M. Bonard, L. Forró, T. Nussbaumer, and C. Schönenberger, Nature (London) 397, 673 (1999).

${ }^{6}$ D. Lovall, M. Buss, E. Graugnard, R. P. Andres, and R. Reifenberger, Phys. Rev. B 61, 5683 (2000).

${ }^{7}$ S. S. Wong, E. Joselevisch, A. T. Woolley, C. L. Cheung, and C. M. Lieber, Nature (London) 394, 52 (1998).

${ }^{8}$ P. Kim and C. M. Lieber, Science 286, 2148 (1999).

${ }^{9}$ M. P. Anantram, Appl. Phys. Lett. 78, 2055 (2001).

${ }^{10}$ E. W. Wong, P. E. Sheehan, and C. M. Lieber, Science 393, 49 (1997).

${ }^{11}$ S. J. Tans, A. R. M. Verschueren, and C. Dekker, Nature (London) 393, 49 (1998).

${ }^{12}$ H. W. Hao, A. M. Baró, and J. J. Sáenz, J. Vac. Sci. Technol. B 9, 1323 (1991); J. Hu, X. D. Xiao, and M. Salmeron, Appl. Phys. Lett. 67, 476 (1995).

${ }^{13}$ A. Bachtold, M. S. Fuhrer, S. Plyasunov, M. Forero, E. H. Anderson, A. Zettl, and P. L. McEuen, Phys. Rev. Lett. 84, 6082 (2000).

${ }^{14}$ M. Burghard, G. Duesberg, G. Philipp, J. Muster, and S. Roth, Adv. Mater. 10, 584 (1998).

${ }^{15}$ P. J. de Pablo, M. T. Martinez, J. Colchero, J. Gómez-Herrero, W. K. Maser, A. M. Benito, E. Muñoz, and A. M. Baró, Adv. Mater. 12, 573 (2000).

${ }^{16}$ H. Dai, E. W. Wong, and C. M. Lieber, Science 272, 523 (1996).

${ }^{17}$ P. J. de Pablo, J. Colchero, M. Luna, J. Gómez-Herrero, and A. M. Baró, Phys. Rev. B 61, 14179 (2000).

${ }^{18} F_{e}$ can be written as $F_{e}=1 / 2 \partial C / \partial z V^{2}$, where $C$ is the capacitance between tip and surface.

${ }^{19}$ When $V_{\text {tip }}=0$ or when $V_{\text {tip }} \neq 0$ and the tip is on top of any connected area to ground, like connected nanotubes and the gold electrode itself (see Fig. $1)$.

${ }^{20} F_{e}\left(z_{0}+\Delta z\right)=F_{e}\left(z_{0}\right)+F_{e}^{\prime}\left(z_{0}\right) \cdot \Delta z+O\left(\Delta z^{2}\right)$. This can be assumed for low interaction regimes.

${ }^{21} A_{\text {set }}$ in the dashed line of Fig. 3.

${ }^{22}$ In order to obtain quantitative data a phase lock loop (PLL) is required. Using PLL the mixture of topography and electrostatic signals can be avoided. Quantitative measurements are currently undertaken.

${ }^{23}$ Work on this issue is currently in progress. 